Padmanabhanunni, A. \& Sui, X-C. (2017). Mental healthcare providers' attitudes towards the adoption of evidence-based practice in the treatment of post-traumatic stress disorder in

\title{
Mental healthcare providers' attitudes towards the adoption of evidence-based practice in the treatment of post-traumatic stress disorder in South Africa
}

\author{
Anita Padmanabhanunni and Xin-Cheng Sui
}

\begin{abstract}
South African society is characterised by a high prevalence of exposure to traumatic events that can lead to post-traumatic stress disorder. In treating post-traumatic stress disorder, mental healthcare providers are tasked with ensuring that their practice is evidence based. However, existing evidence indicates that the rates of adoption of evidence-based practice are generally poor. The promotion and implementation of evidence-based practice into routine clinical settings requires an understanding of the attitudes of mental healthcare providers towards these practices. This study investigated attitudes towards evidence-based practice in the treatment of post-traumatic stress disorder. Participants were 60 mental healthcare providers (clinical/counselling psychologists, social workers, and registered counsellors) in the Western Cape Province working predominantly with trauma survivors. Participants completed a demographic survey and the Attitude Towards Evidence-Based Practice Scale, adapted to assess for attitudes towards the adoption of evidence-based practice in relation to the treatment of post-traumatic stress disorder. Overall, participants reported favourable attitudes towards evidence-based practice in the treatment of posttraumatic stress disorder. Statistical analysis revealed that occupation, age, and gender significantly correlated with attitudes. Social workers and registered counsellors were more likely to report adopting evidence-based practice for post-traumatic stress disorder compared to clinical and counselling psychologists. Older participants and women also reported more favourable attitudes towards evidence-based practice. The implications of these findings are discussed.
\end{abstract}

The evidence-based practice (EBP) movement has been heralded as one of the major advances in health care. EBP is based on the idea that clinical decision-making and practice should be based on rigorous scientific evidence. EBP in psychology (EBPP) is defined as (a) the integration of the best available research evidence (b) with clinical expertise (c) in the context of the patient's values, characteristics, culture, preferences, and circumstances (American Psychological Association, 2010; American Psychological Association Presidential Task Force on Evidence-Based Practice, 2006; McHugh \& Barlow, 2012; Spring, 2007). The shift towards EBPP has been spurred by two converging developments. The first is the rapid advancement in the understanding of the nature of various psychological disorders and the related development of more precisely targeted psychological interventions. The second 
involves the increased emphasis on reducing the burden of mental illness by minimising error in treatment selection and administration (McHugh \& Barlow, 2012). Essentially, by selecting and using interventions with the strongest efficacy, the argument is that clinicians can reduce the amount of time patients spend undergoing treatment, improve clinical outcomes, and maintain high standards of care (Lilienfeld, Ritschel, Lynn, Cautin, \& Latzman, 2013).

This study focused on EBP in the treatment of post-traumatic stress disorder (PTSD), a priority mental disorder in the country (Atwoli et al., 2013; Eagle \& Kaminer, 2014; Edwards, 2013; Kaminer, du Plessis, Hardy, \& Benjamin, 2013). The symptoms of PTSD include intrusive re-experiencing of the traumatic event in the form of nightmares, flashbacks and sensory impressions, cognitive and behavioural attempts to avoid reminders, emotional numbing, amnesia for specific parts of the trauma, and symptoms of physiological hyperarousal (American Psychiatric Association, 2013). Once an individual develops PTSD, the average duration of the disorder without treatment is 42 months (Atwoli et al., 2013). PTSD takes a severe toll on interpersonal relationships and occupational and academic functioning. It is associated with suicidal behaviour, substance abuse problems, and depression (Wilson, Friedman, \& Lindy, 2012).

Various studies in South Africa (Shields, Nadasen, \& Pierce, 2008; Suliman et al., 2009) have attested to the prevalence of PTSD in the general population. According to the South Africa Stress and Health Study (SASHS; Atwoli et al., 2013), which represents the most comprehensive psychiatric epidemiological study conducted to date, over one-third of the adult population in the country has been exposed to some form of criminal victimisation during their lifetime. Lifetime and 12-month prevalence rates of PTSD were $2.3 \%$ and $0.7 \%$, respectively, while the conditional prevalence of PTSD after trauma exposure was $3.5 \%$. Given this context, it is likely that a significant proportion of the population may require psychological treatment, and using evidence-based psychological interventions (EBPIs) to treat PTSD would ensure that trauma survivors have expedient access to the most effective interventions (Edwards, 2005, 2010; Kagee, 2006). However, the transfer of EBPIs from academic research settings to clinical practice has consistently been found to be poor, and the situation in South Africa is no different (Aarons, Glisson et al., 2010; Kazdin, 2014, 2015; Lilienfeld et al., 2013). This was evident in the findings of a study by Higson-Smith, Thacker, and Sikhakhane (2005) involving a needs assessment for traumatic stress services in one of South Africa's provinces. The study is one of the few to provide information about the types of interventions used to treat trauma in local contexts. Data were collected from 214 mental healthcare providers working predominantly with trauma survivors. Participants included psychologists, social workers, and registered counsellors working in non-governmental organisations (NGOs), non-profit organisations, government institutions, and the private sector. A significant finding was that participants reported using general forms of psychotherapy when asked about their work with psychological trauma. Higson-Smith et al. (2005) emphasised that 'not one technique especially associated with traumatic stress work' (p. 34) was reported by these service providers. This suggests that in local contexts, clinical work with trauma does not necessarily incorporate EBP, and various researchers have 
argued that this represents a significant public health concern (Edwards, 2010; Kagee, 2006; Leibowitz-Levy, 2005).

Existing research has found that some of the dominant factors underlying the poor adoption of EBP include knowledge barriers particularly lack of familiarity and training in EBPIs, unfavourable attitudes towards EBP and contextual barriers including lack of institutional support, limited financial resources, and few opportunities for training (Beidas \& Kendall, 2010). Of these factors, attitudes towards EBP have consistently been found to be the most significant predictors of self-reported EBP use among service providers (Aarons, 2004; Borntrager, Chorpita, Higa-McMillan, \& Weisz, 2009).

\section{Mental healthcare provider attitudes towards EBP}

Research on service provider attitudes towards EBP have produced mixed results with some studies indicating positive attitudes (Aarons, 2004), while others indicate unfavourable attitudes (Nakamura, Higa-McMillan, Okamura, \& Shimabukuro, 2011). Individual differences among therapists including age, gender, education, length of time since training, work setting, and therapeutic orientation are some of the factors that have been proposed to account for these varied results. Gray, Elhai, and Schmidt (2007), for example, found that among a sample of professionals who predominantly worked with trauma survivors, younger age and therapeutic orientation was associated with more favourable selfreported attitudes towards EBP. Service providers who endorsed cognitive-behavioural therapy were more likely to report using EBP. Employment in private sector organisations (e.g., NGOs and private clinics) compared to government institutions has also been associated with self-reported positive attitudes towards the adoption of EBP (Aarons \& Sawitzky, 2006). In addition, length of time since training has been shown to have a bearing on attitudes with early career service providers reporting more favourable attitudes towards EBP (Aarons, 2004). Training and supervision in the use of EBPIs has also been associated with increased willingness to adopt EBP (Nakamura et al., 2011). Beliefs about EBP represent another factor that has been shown to influence attitudes (Lilienfeld et al., 2013). Service providers who believe that EBPIs are rigid treatments that hinder clinical creativity and that EBP does not consider the complexity of the client population they serve have been found to report unfavourable attitudes (Lilienfeld et al., 2013). Regardless, the implementation of EBP in real-world settings has been identified (Aarons, Glisson et al., 2010) as imperative in improving the quality of mental health care afforded to vulnerable populations. Given the prevalence of trauma and PTSD in South Africa, it is important for trauma survivors to have access to treatments that have been found to have the greatest efficacy. Attitudes towards EBP have been consistently shown to predict self-reported use of EBP. However, there is limited South African research focusing on these aspects.

The aims of this study were twofold. First, the study aimed to investigate attitudes towards EBP in the treatment of PTSD among mental healthcare providers working with traumatised patients in local contexts. Second, the study aimed to determine whether certain individual (age and gender) and professional factors (number of years in clinical practice, category of registration, and type of work setting) impacted on self-reported attitudes. 


\section{Method}

A cross-sectional survey design was used for the study.

\section{Participants}

The study was conducted in the Western Cape province of South Africa. Participants were 60 mental healthcare providers (i.e., clinical and counselling psychologists, social workers, and registered counsellors) working specifically in the area of trauma and PTSD. These categories of service providers were selected because they are more likely to be at the front lines in terms of the provision of psychological care to trauma survivors.

Table I. Participant demographics.

\begin{tabular}{llcl}
\hline Demographics & & $N$ & Percentage \\
\hline Gender & Female & 44 & 73.3 \\
Race & Male & 16 & 26.7 \\
& White & 40 & 66.7 \\
& Coloured & 9 & 15.0 \\
& Black & 4 & 6.7 \\
& Indian & 4 & 6.7 \\
Occupation & Other & 3 & 5.0 \\
& Counselling & 20 & 33.3 \\
Work setting & Clinical & 27 & 45.0 \\
& Other & 13 & 21.7 \\
& Private practice & 32 & 53.3 \\
Age (years) & Hospital/clinic & 13 & 21.7 \\
Years in clinical practice & Academic & 8 & 13.3 \\
Patients with PTSD per month & NGO & 7 & 11.7 \\
\hline
\end{tabular}

NGO: non-governmental organisation; PTSD: post-traumatic stress disorder; SD: standard deviation.

Inclusion criteria for participation in the study were (a) clinical work primarily with survivors of trauma, (b) seeing a minimum of two clients with PTSD per month, and (c) registration with their regulatory body. Psychologists were identified through open-access psychology websites (e.g., http://www.psychotherapy.co.za/) where providers advertise psychological services and indicate their area of interest (e.g., the types of psychological conditions or population group that they work with). This allowed for the targeting of practitioners who specifically work with trauma. NGOs and clinics providing trauma counselling were also contacted. Through this process, a list of 75 service providers who work mostly with survivors of trauma was generated. These service providers were subsequently contacted via email and by telephone and provided with information regarding the research project and a request to participate. A total of 60 responded and were included in the study. The response rate was $80 \%$. 
With regard to demographics (Table 1), the majority of participants were women (73.3\%). Most participants described themselves as White (66.7\%), followed by Coloured (15.0\%), Indian (6.7\%), and Black (6.7\%). In terms of occupation, the majority were clinical psychologists (45\%), followed by counselling psychologists (33.3\%) and social workers and registered counsellors (21.7\%). Average number of years in clinical practice was nine. Participants ranged in age from 23 to 77 years $(M=42$ years). The majority of participants worked in private practice settings (53.3\%). Other work settings included psychiatric hospitals (21.7\%), academic settings (13.3\%), and NGOs (11.7\%). Participants saw an average of three clients with PTSD per month with a maximum of 12 PTSD clients seen per month.

\section{Instruments}

The electronic survey consisted of a demographic questionnaire and Aarons' (2005) Attitude Towards Evidence-Based Practice Scale (EBPAS). The demographic questionnaire included questions pertaining to participant's age, work setting, number of years in clinical practice, types of traumatic events encountered in their clinical work, and average number of patients with PTSD treated per month.

The EBPAS is a 15 -item measure that assesses general attitudes towards EBP. It comprises four subscales: appeal to EBP (e.g., 'If you received training in a therapy or intervention that was new to you, how likely would you be to adopt it if it made sense to you?'), requirements for the use of EBP (e.g., 'If you received training in a therapy or intervention that was new to you, how likely would you be to adopt it if it was mandated by your regulatory body?'), openness to innovation (e.g., 'I am willing to try new types of therapy or interventions even if I have to follow a treatment manual?'), and divergence with clinical practice (e.g., 'Clinical experience is more important than using manualised therapy or interventions'; Aarons, 2005). Participants indicate their level of agreement with each item with responses ranging from $0=$ not at all to $4=$ to a great extent .

With consent from the designer, this scale was adapted to specifically focus on EBP in the treatment of PTSD and piloted among 15 service providers. In the original version, questions focused on attitudes towards EBP broadly as can be seen in the examples above. In the adapted version, the wording of the items was changed to specifically focus on PTSD (e.g., 'If you received training in a therapy or intervention for PTSD that was new to you, how likely would you be to adopt it if it made sense to you?' and 'I am willing to try new types of therapy or interventions for PTSD even if I have to follow a treatment manual?'). The EBPAS has been used in culturally diverse contexts (Majid et al., 2011) apart from which it was designed and has been found to have sound content validity and internal consistency (Cronbach's alphas ranged from .67 to .89 for subscales with an overall scale alpha of .72; van Sonsbeek et al., 2015).

\section{Procedure}

An online survey was generated using Google Forms, and the link to the survey was distributed to participants via email. Responses to the survey were stored on the second author's (X.-C.S) Google Drive. 


\section{Ethical considerations}

Ethical approval for the study was obtained from the University of the Western Cape through the Department of Psychology. All participants provided informed consent before completing the survey. The online survey was anonymous.

\section{Data analysis}

The demographic data were organised using an Excel spread sheet and analysed manually and evaluated by an independent assessor to ensure rigour. Data from the EBPAS were captured and scored using the Statistical Package for the Social Sciences (SPSS) software (IBM SPSS statistics 23). Correlational analysis was conducted for continuous variables (i.e., age and years in clinical practice) to determine whether these factors related to attitudes. A one-way multivariate analysis of variance (MANOVA) was conducted for categorical variables, namely, gender, type of work setting, and occupation.

\section{Results}

Demographic information is presented in Table 1. With regard to trauma work, participants reported that the most common types of trauma they encountered in their clinical work included sexual assault (40\%), robbery-related trauma (e.g., hijacking and armed robbery; 23.3\%), neglect and physical abuse during childhood (10\%), domestic violence (5\%), community violence (5\%), and work-related trauma (e.g., where trauma survivors were policemen, fire fighters, or worked in the military; 1.7\%). Descriptive statistics and Cronbach's alpha for the EBPAS are provided in Table 2.

Table 2. Descriptive statistics and Cronbach's alpha for the EBPAS.

\begin{tabular}{llll}
\hline Subscale & $M$ & SD & Alpha \\
\hline Requirements & 2.20 & 1.06 & .85 \\
Appeal & 2.93 & 0.63 & .72 \\
Openness & 2.46 & 0.83 & .86 \\
Divergence & 2.80 & 0.78 & .66 \\
EBPAS total & 2.62 & 0.53 & .80 \\
\hline
\end{tabular}

EBPAS: Attitude Towards Evidence-Based Practice Scale; SD: standard deviation.

Table 3. The relationship between age, years in practice, and EBPAS.

\begin{tabular}{llll}
\hline EBPAS & & Age & Years in practice \\
\hline Requirement & Pearson correlation & -.135 & -.115 \\
Appeal & Pearson correlation & -.080 & -.081 \\
Openness & Pearson correlation & $.263^{*}$ & .192 \\
Divergence & Pearson correlation & -.158 & -.139 \\
EBPAS total & Pearson correlation & -.032 & -.046 \\
\hline
\end{tabular}

EBPAS: Attitude Towards Evidence-Based Practice Scale. $*_{p}<.05$. 
Regarding the EBPAS, subscale alphas ranged from .66 to .86 with an overall scale alpha of .80 indicating high internal consistency and scale reliability. Overall, respondents reported a mean score of 2.62 on the EBPAS, indicating a more global positive attitude towards EBP in the treatment of PTSD. The appeal subscale had the highest mean (2.93), which means that participants reported that they would be more likely to adopt an EBP for PTSD if it 'made sense' to them, if they knew how to use it correctly, and if it was being used by a colleague who was happy with it. The divergence subscale had the second highest mean (2.80), indicating that participants reported viewing clinical experience as more important in treating PTSD compared to manualised treatments. The requirement subscale had the lowest mean (2.20). This means that participants reported that they would be less likely to adopt an EBPI for PTSD if it was mandated by their regulatory body.

According to Table 3, there was a significant positive relationship between age and reported openness to adopting EBP $(r=.263, p<.05)$. Older participants reported an increased likelihood of adopting EBP in the treatment of PTSD compared to their younger counterparts. No significant relationship was found between years in clinical practice and attitudes.

As shown in Table 4, the multivariate statistics was significant for gender (Pillai's trace $=$ $2.91, p<.05$ ) and occupation (Pillai's trace $=2.99, p<.01$ ). Univariate analysis indicated a significant difference between women and men in terms of the requirements subscale $(F=$ 8.33, $p<.01)$ and the total EBPAS $(F=5.89, p<.05)$ with women scoring significantly higher compared to men on these two scales. This means that women reported more favourable attitudes towards EBP in the treatment PTSD and more likely to adopt EBP for PTSD if it was mandated. Multivariate statistics was also significant for occupation (Pillai's trace $=2.99, p<$ .01). Post hoc analysis indicated that social workers and registered counsellors scored higher on the requirements subscale compared to clinical $(p<.01)$ and counselling $(p<.01)$ psychologists. No significant relationship was found between type of work setting and attitudes (Table 4). 
Table 4. Results of MANOVA for EBPAS subscales.

\begin{tabular}{|c|c|c|c|c|c|c|c|c|c|c|c|c|c|c|c|}
\hline & \multicolumn{3}{|c|}{ Requirements } & \multicolumn{3}{|c|}{ Appeal } & \multicolumn{3}{|c|}{ Openness } & \multicolumn{3}{|c|}{ Divergence } & \multicolumn{3}{|c|}{ EBPAS total } \\
\hline & $M$ & $S D$ & $F$ & $M$ & $S D$ & $F$ & M & $S D$ & $F$ & M & $S D$ & $F$ & M & $S D$ & $F$ \\
\hline $\begin{array}{l}\text { Gender (Pillai's } \\
\text { trace }=\left.2.9\right|^{*} \text { ) }\end{array}$ & & & $8.33^{\text {tok }}$ & & & 1.90 & & & 0.14 & & & 2.90 & & & $5.89 *$ \\
\hline Male & & 1.58 & 1.10 & & 2.75 & 0.63 & & 2.39 & 0.79 & & 2.52 & 0.99 & & 2.36 & 0.60 \\
\hline Female & & 2.42 & 0.96 & & 3.00 & 0.62 & & 2.48 & 0.85 & & 2.90 & 0.67 & & 2.72 & 0.47 \\
\hline $\begin{array}{l}\text { Occupation (Pillai's } \\
\text { trace }=2.99^{* * *} \text { ) }\end{array}$ & & & $9.07^{* * k}$ & & & 0.04 & & & 0.04 & & & 1.16 & & & 1.14 \\
\hline Counselling & 1.95 & 1.15 & & 2.96 & 0.72 & & 2.44 & 0.99 & & 3.01 & 0.66 & & 2.63 & 0.54 & \\
\hline Clinical & 1.91 & 0.89 & & 2.93 & 0.62 & & 2.44 & 0.84 & & 2.69 & 0.88 & & 2.53 & 0.59 & \\
\hline Other & & 3.18 & 0.59 & & 2.90 & 0.51 & & 2.52 & 0.56 & & 2.69 & 0.72 & & 2.80 & 0.34 \\
\hline $\begin{array}{l}\text { Setting (Pillai's } \\
\text { trace }=1.18 \text { ) }\end{array}$ & & & 2.63 & & & 0.26 & & & 1.00 & & & 0.71 & & & 0.64 \\
\hline Private practice & & 2.01 & 1.12 & & 2.94 & 0.68 & & 2.29 & 0.94 & & 2.83 & 0.81 & & 2.55 & 0.61 \\
\hline Hospital/clinic & & 2.10 & 0.67 & & 2.85 & 0.55 & & 2.71 & 0.75 & & 2.71 & 0.72 & & 2.63 & 0.43 \\
\hline Academic & 2.25 & 1.07 & & 2.91 & 0.63 & & 2.63 & 0.52 & & 3.06 & 1.00 & & 2.74 & 0.54 & \\
\hline NGO & & 3.19 & 0.98 & & 3.11 & 0.57 & & 2.57 & 0.66 & & 2.50 & 0.38 & & 2.82 & 0.27 \\
\hline
\end{tabular}

MANOVA: multivariate analysis of variance; EBPAS: Attitude Towards Evidence-Based Practice Scale; SD: standard deviation; NGO: non-governmental organisation.

$*_{k} p<.01, * p<.05$.

\section{Discussion}

The aim of this study was to investigate the attitudes of mental healthcare providers towards EBP in the treatment of PTSD in South Africa. Overall, service providers reported favourable attitudes with regard to adopting evidence-based interventions in treating trauma survivors. This finding corresponds to the existing literature on the self-reported general attitudes of service providers (Aarons \& Palinkas, 2007; Brown, Wickline, Ecoff, \& Glaser, 2009; Salbach, Jaglal, Korner-Bitensky, Rappolt, \& Davis, 2007). Also, similar to existing research (Aarons \& Sawitzky, 2006; McKee, 2014) was the finding that occupation impacted on attitudes with social workers and registered counsellors reporting greater willingness to adopt EBP for PTSD if it was mandated by their regulatory body.

In contrast with the literature, this study found that older service providers reported more favourable attitudes towards EBP for PTSD. The majority of existing research has found the opposite with positive attitudes towards EBP being held by younger practitioners (Aarons, Sommerfeld, \& Walrath-Greene, 2009; Gray et al., 2007; Jette et al., 2003). A possible explanation for this finding is that older service providers may have found that the typical interventions they have been using do not yield the desired results thereby motivating the move to EBP. Another explanation is that learning and implementing EBP confers certain professional advantages including enhanced sense of competence, and this may have influenced their attitudes. Alternatively, social desirability bias may also account for the above finding. 
Table 5. Multiple comparisons between professional categories and the EBPAS.

\begin{tabular}{|c|c|c|c|c|c|c|c|}
\hline \multicolumn{8}{|l|}{ Bonferroni } \\
\hline \multirow[t]{2}{*}{$\begin{array}{l}\text { Dependent } \\
\text { variable }\end{array}$} & \multirow{2}{*}{$\begin{array}{l}\text { (l) } \\
\text { Professional } \\
\text { category }\end{array}$} & \multirow[t]{2}{*}{$\begin{array}{l}\text { (J) Professional } \\
\text { category }\end{array}$} & \multirow{2}{*}{$\begin{array}{l}\text { Mean } \\
\text { difference } \\
(I-J)\end{array}$} & \multirow[t]{2}{*}{ SE } & \multirow[t]{2}{*}{ Significance } & \multicolumn{2}{|c|}{$\begin{array}{l}95 \% \text { Confidence } \\
\text { interval }\end{array}$} \\
\hline & & & & & & $\begin{array}{l}\text { Lower } \\
\text { bound }\end{array}$ & $\begin{array}{l}\text { Upper } \\
\text { bound }\end{array}$ \\
\hline \multirow[t]{6}{*}{ Requirements } & \multirow{2}{*}{$\begin{array}{l}\text { Counselling } \\
\text { psychologist }\end{array}$} & Clinical psychologist & .0364 & .27661 & 1.000 & -0.6459 & 0.7187 \\
\hline & & Other & $-1.2295^{* * k}$ & .33403 & 0.002 & -2.0534 & -0.4055 \\
\hline & \multirow{2}{*}{$\begin{array}{l}\text { Clinical } \\
\text { psychologist }\end{array}$} & Counselling psychologist & -.0364 & .27661 & 1.000 & -0.7187 & 0.6459 \\
\hline & & Other & $-1.2659^{*} \%$ & .31651 & 0.001 & -2.0466 & -0.4852 \\
\hline & \multirow[t]{2}{*}{ Other } & Counselling psychologist & $1.2295^{\text {*ak }}$ & .33403 & 0.002 & 0.4055 & 2.0534 \\
\hline & & Clinical psychologist & I.2659 kok & .31651 & 0.001 & 0.4852 & 2.0466 \\
\hline \multirow[t]{6}{*}{ Appeal } & \multirow{2}{*}{$\begin{array}{l}\text { Counselling } \\
\text { psychologist }\end{array}$} & Clinical psychologist & .0366 & .18772 & 1.000 & -0.4265 & 0.4996 \\
\hline & & Other & .0587 & .22669 & 1.000 & -0.5005 & 0.6178 \\
\hline & \multirow{2}{*}{$\begin{array}{l}\text { Clinical } \\
\text { psychologist }\end{array}$} & Counselling psychologist & -.0366 & .18772 & 1.000 & -0.4996 & 0.4265 \\
\hline & & Other & .0221 & .21480 & 1.000 & -0.5078 & 0.5519 \\
\hline & \multirow[t]{2}{*}{ Other } & Counselling psychologist & -.0587 & .22669 & 1.000 & -0.6178 & 0.5005 \\
\hline & & Clinical psychologist & -.0221 & .21480 & 1.000 & -0.5519 & 0.5078 \\
\hline \multirow[t]{6}{*}{ Openness } & \multirow{2}{*}{$\begin{array}{l}\text { Counselling } \\
\text { psychologist }\end{array}$} & Clinical psychologist & -.0069 & .24894 & 1.000 & -0.6210 & $0.607 \mid$ \\
\hline & & Other & -.0817 & .30061 & 1.000 & -0.8233 & 0.6598 \\
\hline & \multirow{2}{*}{$\begin{array}{l}\text { Clinical } \\
\text { psychologist }\end{array}$} & Counselling psychologist & .0069 & .24894 & 1.000 & $-0.607 \mid$ & 0.6210 \\
\hline & & Other & -.0748 & .28485 & 1.000 & -0.7774 & 0.6278 \\
\hline & \multirow[t]{2}{*}{ Other } & Counselling psychologist & .0817 & .30061 & 1.000 & -0.6598 & 0.8233 \\
\hline & & Clinical psychologist & .0748 & .28485 & 1.000 & -0.6278 & 0.7774 \\
\hline \multirow[t]{6}{*}{ Divergence } & \multirow{2}{*}{$\begin{array}{l}\text { Counselling } \\
\text { psychologist }\end{array}$} & Clinical psychologist & .3273 & .22961 & 0.478 & -0.2391 & 0.8937 \\
\hline & & Other & .3202 & .27728 & 0.759 & -0.3638 & 1.0042 \\
\hline & \multirow{2}{*}{$\begin{array}{l}\text { Clinical } \\
\text { psychologist }\end{array}$} & Counselling psychologist & -.3273 & .22961 & 0.478 & -0.8937 & 0.2391 \\
\hline & & Other & $-.007 \mid$ & .26274 & 1.000 & -0.6552 & 0.6410 \\
\hline & \multirow[t]{2}{*}{ Other } & Counselling psychologist & -.3202 & .27728 & 0.759 & -1.0042 & 0.3638 \\
\hline & & Clinical psychologist & $.007 \mid$ & .26274 & 1.000 & -0.6410 & 0.6552 \\
\hline \multirow[t]{6}{*}{ EBPAS_mean } & \multirow{2}{*}{$\begin{array}{l}\text { Counselling } \\
\text { psychologist }\end{array}$} & Clinical psychologist & .1025 & .15622 & 1.000 & -0.2829 & 0.4878 \\
\hline & & Other & -.1667 & .18865 & 1.000 & -0.6320 & 0.2987 \\
\hline & \multirow{2}{*}{$\begin{array}{l}\text { Clinical } \\
\text { psychologist }\end{array}$} & Counselling psychologist & -.1025 & .15622 & 1.000 & -0.4878 & 0.2829 \\
\hline & & Other & -.2691 & .17875 & 0.413 & -0.7101 & 0.1718 \\
\hline & \multirow[t]{2}{*}{ Other } & Counselling psychologist & .1667 & .18865 & 1.000 & -0.2987 & 0.6320 \\
\hline & & Clinical psychologist & .2691 & .17875 & 0.413 & -0.1718 & 0.7101 \\
\hline
\end{tabular}

EBPAS: Attitude Towards Evidence-Based Practice Scale; SE: standard error. $* k_{p}<.01, * p<.05$.

A second noteworthy finding is that work setting had no influence on reported attitudes. Favourable attitudes towards EBP for PTSD were present regardless of the setting in which participants worked. Prior studies (Aarons \& Sawitzky, 2006) have found that mental healthcare providers working in the private sector reported more favourable attitudes towards EBP compared to those who were employed in the government sector. This has been attributed to greater support within private sector organisations for EBP and a facilitative implementation culture (Aarons \& Sawitzky, 2006). However, the majority of participants in this study worked in private practice settings, and this implies that reported interest in adopting EBP for PTSD was not related to the presence of a supportive 
organisational culture. It is probable that other factors such as individual receptivity to EBP had a more over-riding influence on reported attitudes.

A third distinctive finding of the study was that gender significantly correlated with attitudes, with women reporting more favourable attitudes towards EBP for PTSD and more likely to adopt EBP for PTSD if it was mandated by their regulatory body. Existing studies have noted no significant gender differences in attitudes (Aarons, James et al., 2010; Borntrager et al., 2009; McKee, 2014). The reasons for the evidenced gender differences in the study remain unclear. However, since the majority of service providers in the mental health and social service workforce are women, greater attention needs to be given to uncovering the reasons for these differences so as to promote the adoption of EBP across genders.

This study has certain limitations. The EBPAS was a self-administered questionnaire and responses may not be an entirely accurate representation of attitudes, and the possibility of social desirability bias needs to be considered. It is also possible that practitioners with more positive attitudes towards EBP and who are skilled in the use of EBPIs may have been more likely to respond to the request to participate in the study. Furthermore, only participants who advertised their services online or who were employed at sites that specifically cater to trauma survivors were included in the study, and this may have contributed to a sampling bias.

\section{Conclusion}

This study provides important insights into the self-reported attitudes of mental healthcare providers towards adopting EBP in the treatment of PTSD. The majority of service providers reported being favourably disposed towards using the evidence from empirical research to inform their clinical practice. Given this, it would be important to create opportunities to train practitioners in the use of efficacious interventions for addressing trauma and PTSD.

\section{Funding}

This work was financially supported by the National Research Foundation (NRF) of South Africa. 


\section{References}

Aarons, G. A. (2004). Mental health provider attitudes toward adoption of evidence-based practice: The Evidence-Based Practice Attitude Scale (EBPAS). Mental health services research, 6(2), 61-74.

Aarons, G. A. (2005). Measuring provider attitudes toward evidence-based practice: Consideration of organizational context and individual differences. Child and Adolescent Psychiatric Clinics of North America, 14, 255-271.

Aarons, G. A., Glisson, C., Hoagwood, K., Kelleher, K., Landsverk, J., \& Cafri, G. (2010). Psychometric properties and US National norms of the Evidence-Based Practice Attitude Scale (EBPAS). Psychological Assessment, 22, 356-365.

Aarons, G. A., James, S., Monn, A. R., Raghavan, R., Wells, R. S., \& Leslie, L. K. (2010). Behavior problems and placement change in a national child welfare sample: A prospective study. Journal of the American Academy of Child \& Adolescent Psychiatry, 49, 70-80.

Aarons, G. A., \& Palinkas, L. A. (2007). Implementation of evidence-based practice in child welfare: Service provider perspectives. Administration and Policy in Mental Health and Mental Health Services Research, 34, 411-419.

Aarons, G. A., \& Sawitzky, A. C. (2006). Organizational culture and climate and mental health provider attitudes toward evidence-based practice. Psychological Services, 3, $61-72$.

Aarons, G. A., Sommerfeld, D. M., \& Walrath-Greene, C. M. (2009). Evidence-based practice implementation: The impact of public versus private sector organization type on organizational support, provider attitudes, and adoption of evidence-based practice. Implementation Science, 4, 83.

American Psychiatric Association. (2013). Diagnostic and statistical manual of mental disorders (5th ed.). Arlington, VA: American Psychiatric Publication.

American Psychological Association. (2010). Ethical principles of psychologists and code of conduct: Including 2010 amendments. Washington, DC: Author.

American Psychological Association Presidential Task Force on Evidence-Based Practice. (2006). Evidence-based practice in psychology. American Psychologist, 61, 271-285.

Atwoli, L., Stein, D. J., Williams, D. R., Mclaughlin, K. A., Petukhova, M., Kessler, R. C., \& Koenen, K. C. (2013). Trauma and posttraumatic stress disorder in South Africa: Analysis from the South African Stress and Health Study. BMC Psychiatry, 13, 182.

Beidas, R. S., \& Kendall, P. C. (2010). Training therapists in evidence-based practice: A critical review of studies from a systems-contextual perspective. Clinical Psychology: Science and Practice, 17, 1-30.

Borntrager, C. F., Chorpita, B. F., Higa-McMillan, C., \& Weisz, J. R. (2009). Provider attitudes toward evidence-based practices: Are the concerns with the evidence or with the manuals? Psychiatric Services, 6o, 677-681.

Brown, C. E., Wickline, M. A., Ecoff, L., \& Glaser, D. (2009). Nursing practice, knowledge, attitudes and perceived barriers to evidence-based practice at an academic medical center. Journal of Advanced Nursing, 65, 371-381.

Eagle, G. T., \& Kaminer, D. (2014). Traumatic stress: Established knowledge, current debates and new horizons. South African Journal of Psychology, 45, 22-35. 
Edwards, D. (2005). Post-traumatic stress disorder as a public health concern in South Africa. Journal of Psychology in Africa, 15, 125-134.

Edwards, D. J. (2010). Using systematic case studies to investigate therapist responsiveness: Examples from a case series of PTSD treatments. Pragmatic Case Studies in Psychotherapy, 6, 255-275.

Edwards, D. J. (2013). Responsive integrative treatment of clients with PTSD and traumarelated disorders: An expanded evidence-based model. Journal of Psychology in Africa, 23, 7-19.

Gray, M. J., Elhai, J. D., \& Schmidt, L. O. (2007). Trauma professionals' attitudes toward and utilization of evidence-based practices. Behavior Modification, 31, 732-748.

Higson-Smith, C., Thacker, M., \& Sikhakhane, N. (2005). Needs assessment for integrated traumatic stress services: Mpumalanga Province, South Africa. Mpumalanga, South African Institute for Traumatic Stress and Themba Lesizwe. Retrieved from http://wiredspace.wits.ac.za/jspui/bitstream/10539/13163/1/

INTERPROFESSIONAL\%20COMMUNICATION\%2OIN\%2OA\%2ORURAL\%2OHOS PITAL\%20-\%20C.\%2OLONGMAN.pdf

Jette, D. U., Bacon, K., Batty, C., Carlson, M., Ferland, A., Hemingway, R. D., \& Volk, D. (2003). Evidence-based practice: Beliefs, attitudes, knowledge, and behaviours of physical therapists. Physical Therapy, 83, 786-805.

Kagee, A. (2006). Where is the evidence in South African clinical psychology? South African Journal of Psychology, 26, 233-248.

Kaminer, D., du Plessis, B., Hardy, A., \& Benjamin, A. (2013). Exposure to violence across multiple sites among young South African adolescents. Peace and Conflict: Journal of Peace Psychology, 19(2), 112.

Kazdin, A. E. (2014). Evidence-based psychotherapies I: Qualifiers and limitations in what we know. South African Journal of Psychology, 44, 381-403.

Kazdin, A. E. (2015). Evidence-based psychotherapies II: Changes in models of treatment and treatment delivery. South African Journal of Psychology, 45, 3-21.

Leibowitz-Levy, S. (2005). The role of brief term interventions with South African child trauma survivors. Journal of Psychology in Africa, 15, 155-163.

Lilienfeld, S. O., Ritschel, L. A., Lynn, S. J., Cautin, R. L., \& Latzman, R. D. (2013). Why many clinical psychologists are resistant to evidence-based practice: Root causes and constructive remedies. Clinical Psychology Review, 33, 883-890.

Majid, S., Foo, S., Luyt, B., Xue, Z., Yin-Leng, T., Yun-Ke, C., \& Mokhtar, I. A. (2011). Adopting evidence-based practice in clinical decision making: Nurses' perceptions, knowledge, and barriers. Journal of the Medical Library Association, 99, 229-236.

McHugh, R. K., \& Barlow, D. H. (Eds.). (2012). Dissemination and implementation of evidence-based interventions. New York: Oxford University Press.

McKee, E. (2014). Evidence-based practice attitudes, knowledge and perceptions of barriers among juvenile justice professionals. Retrieved from http://scholarcommons.usf.edu/cgi/viewcontent.cgi?article=6467\&; context=etd

Nakamura, B. J., Higa-McMillan, C. K., Okamura, K. H., \& Shimabukuro, S. (2011). Knowledge of and attitudes towards evidence-based practices in community child 
mental health practitioners. Administration and Policy in Mental Health and Mental Health Services Research, 38, 287-300.

Salbach, N. M., Jaglal, S. B., Korner-Bitensky, N., Rappolt, S., \& Davis, D. (2007). Practitioner and organizational barriers to evidence-based practice of physical therapists for people with stroke. Physical Therapy, 87, 1284-1303.

Shields, N., Nadasen, K., \& Pierce, L. (2008). The effects of community violence on children in Cape Town, South Africa. Child Abuse \& Neglect: The International Journal, 32, 589-601.

Spring, B. (2007). Evidence-based practice in clinical psychology: What it is; why it matters; what you need to know. Journal of Clinical Psychology, 63, 611-631.

Suliman, S., Mkabile, S. G., Fincham, D. S., Ahmed, R., Stein, D. J., \& Seedat, S. (2009). Cumulative effect of multiple trauma on symptoms of PTSD, anxiety and depression in adolescents. Comprehensive Psychiatry, 5O, 121-127.

van Sonsbeek, M. A., Hutschemaekers, G. J., Veerman, J. W., Kleinjan, M., Aarons, G. A., \& Tiemens, B.

G. (2015). Psychometric properties of the Dutch version of the Evidence-Based Practice Attitude Scale (EBPAS). Health Research Policy and Systems, 13, 69.

Wilson, J. P., Friedman, M. J., \& Lindy, J. D. (2012). Treating psychological trauma and PTSD. New York: Guilford Press. 